\title{
Lyotropic nematics : molecular aggregation and susceptibilities
}

\author{
J. Charvolin, A. M. Levelut \\ Laboratoire de Physique des Solides $\left(^{*}\right)$, Bât. 510, Université Paris-Sud, 91405 Orsay, France
}

and E. T. Samulski (**)

Department of Chemistry and Institute of Materials Science, University of Connecticut, Storrs, CT 06268, U.S.A.

(Reçu le 6 août 1979, accepté le 28 septembre 1979)

\begin{abstract}
Résumé. - Une variation de la concentration d'un composant dans une phase nématique lyotrope peut changer le signe de l'anisotropie de sa susceptibilité magnétique $\Delta \chi$. Le signe de $\Delta \chi$ est déterminé par l'observation optique des textures induites par un champ magnétique. La diffraction des rayons $\mathrm{X}$ permet une première approche du mode d'agrégation des molécules amphiphiles. Nous concluons que l'inversion de $\Delta \chi$ provient d'un changement de forme des agrégats, sans qu'il y ait inversion du signe du tenseur de susceptibilité moléculaire.
\end{abstract}

Abstract. - Alterations of the concentrations of certain components in a lyotropic nematic mesophase may change the sign of the anisotropy of its magnetic susceptibility, $\Delta \chi$. We report here : 1) the determination of the sign of $\Delta \chi$ by optical observation of the textures in the presence of a magnetic field, 2) the results of an X-ray diffraction study of the aggregation of the amphiphilic molecules. We conclude that the inversion of $\Delta \chi$ results from the different shapes of the aggregates.

Introduction. - Lyotropic nematic phases, mixtures of surfactants, long chain alcohols and water (with or without salts) are anisotropic fluids which may behave as ordinary thermotropic nematics do [1, 2] : field,

1) they orient spontaneously in a strong magnetic

2) they may exhibit thread-like textures.

These phases are frequently being used as orienting media in NMR determination of the structures of dissolved molecules [3] with the advantage, when compared to thermotropic phases, that they can dissolve molecules with a range of polarities, since they consist of two media, aqueous and paraffinic. Ferrofluids also have been dissolved in these phases enabling the measurement of magneto-optical couplings with weak magnetic fields [4, 5].

A dramatic and intrinsic property of the lyotropic nematic phases is the change in sign of the magnetic susceptibility $\Delta \chi$ anisotropy induced by changes of the relative concentration of their components [6]. As they are lyotropic phases their structural elements

(*) Laboratoire associé au C.N.R.S.

(**) On sabbatic leave. are most likely aggregates of amphiphilic molecules [7], not isolated molecules as in the thermotropic phases, and an important point therefore is to determine whether the inversion of $\Delta \chi$ can be attributed to either a change of sign of the molecular susceptibility tensor or to a change of the aggregation state. We have undertaken a structural study of those phases by X-ray diffraction and we present here preliminary results and conclusions.

Samples. - Samples are prepared from the now classical mixture water, sodium decyl sulfate (hereafter called SDS), decanol [1]. It has been shown [6] that, at $27^{\circ} \mathrm{C}$, when the concentrations in weight are $53 \% \mathrm{H}_{2} \mathrm{O}, 40 \%$ SDS, $7 \%$ decanol, the phase has a positive anisotropy of susceptibility $\Delta \chi>0$ (the phase is said to be of type I); when more than $0.3 \% \mathrm{SO}_{4} \mathrm{Na}_{2}$ is added the phase turns to a phase with $\Delta \chi<0$ (it is said to be of type II). Two methods have been used to ascertain the sign of $\Delta \chi, \mathrm{NMR}$ and microscopy.

NuClear magnetic RESONANCE OF $\mathrm{D}_{2} \mathrm{O}$. - This is the routine, but somewhat cumbersome, way to characterize the type of the phase. Its description 
can be found in [3]; we shall recall the essentials of it briefly. A sample (with $\mathrm{D}_{2} \mathrm{O}$ ) is introduced in the NMR spectrometer. The first spectrum has the usual powder pattern appearance which indicates that the directors $\mathbf{n}$ of the monodomains are randomly oriented. Several minutes later the lines narrow and the powder spectrum evolves into the spectrum of a sample with uniaxial symmetry. The sample has oriented itself in the magnetic field, all directors $\mathbf{n}$ are alined. The optic axis of the sample is either parallel or perpendicular to the field $\mathbf{H}_{0}$ thus revealing the type of the sample : a sample with $\Delta \chi>0\left(\mathbf{n} / / \mathbf{H}_{0}\right)$ is called type I, for $\Delta \chi<0\left(\mathbf{n} \perp \mathbf{H}_{0}\right)$ it is type II. The NMR method is not easy to handle when the viscosity of the sample is so low that its orienting rate is too high compared to the time required to record a spectrum with conventional, non-F.T., spectrometers.

OPTICAL OBSERVATIONS. - The lyotropic nematic phase is smeared between two glass plates, the thickness of the sample being about $0.15 \mathrm{~mm}$. Planar

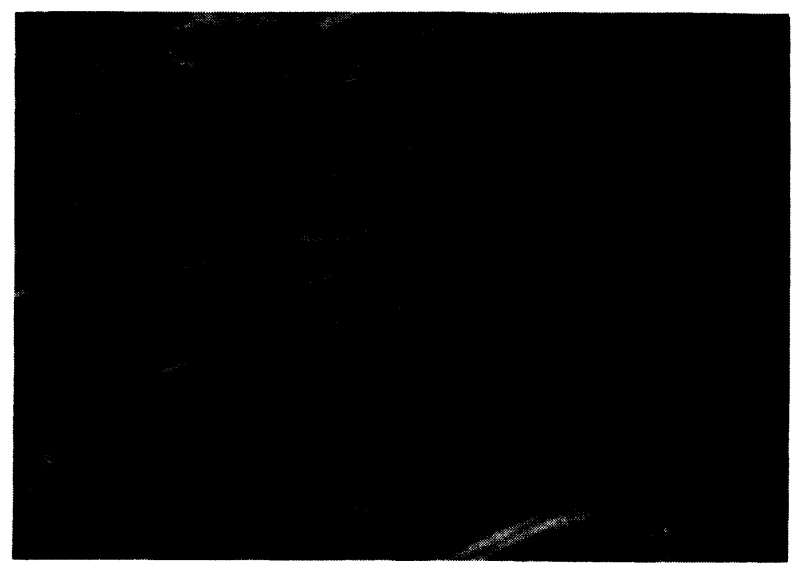

a - type $I(\Delta x>0)$

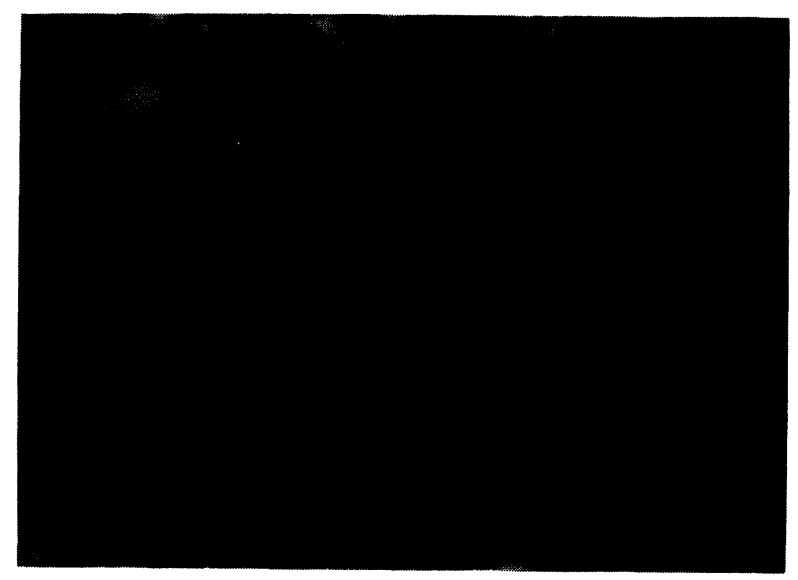

b. type $\mathbb{I}(\Delta x<0)$

Fig. 1. - Textures observed in planar domains of type I $(a)$ and homeotropic domains of type II (b) after application of a $15 \mathrm{kG}$ magnetic field normal to the preparations for a few minutes. The samples thickness is $0.1 \mathrm{~mm}$, but similar pattern can also be seen with $1 \mathrm{~mm}$. One large division of the scale is $100 \mu$. domains, where the director $\mathbf{n}$ is parallel to the glass plates, and homeotropic ones, where $\mathbf{n}$ is normal to the plates, are easily observable. As a matter of fact the full stage may appear homeotropic with type II samples. A magnetic field applied normal to the glass plates will tend to aline the directors of the planar domains of type I along the normal because $\Delta \chi$ is positive ; for type II the directors of the homeotropic domains will depart from the normal because $\Delta \chi$ is negative (a field normal to the glass plates will, of course, have no effect on the type I homeotropic and type II planar domains). Apparently periodic organizations of the isoclines are then observable and the associated textures are shown in figure 1. Each texture is typical of one type of sample. The textural organization develops along one direction for type I because the directors rotate in the plane defined by the original orientation of $\mathbf{n}$ in the planar domain and the magnetic field $\mathbf{H}_{0}$. The organization is two dimensional for type II because the directors reorient away from their original orientation in the homeotropic domains isotropically. The origin of these textures is not yet clear [8].

X-ray diffraction patterns. - X-ray diffraction patterns were obtained with a photographic monochromatic Laue camera [9] with the $\mathrm{CuK}_{\alpha}$ wavelength $\lambda=1.54 \AA$ and a sample to film distance of $80 \mathrm{~mm}$. The samples were in sealed glass capillaries, held perpendicular to the X-ray beam, and oriented by the magnetic field of a $12 \mathrm{kG}$ permanent magnet in the equatorial plane. The diffraction patterns are obtained for two configurations :

1) X-ray beam perpendicular to the director $\mathbf{n}$, with $H_{0}$ present (perpendicular).

2) X-ray beam parallel to the director $\mathbf{n}$, with $H_{0}$ absent (parallel). The results will be discussed as a function of the scattering vector $s$ of the Bragg relation, $|\mathbf{s}|=2 \sin \theta / \lambda$, where $2 \theta$ is the angle between the incident and diffracted beams.

As is currently the case for lyotropic liquid crystals the diffraction patterns can be analyzed in two regions [7] :

1) high angles $\left(s \simeq(5.5 \AA)^{-1}\right)$, where the diffraction is due to the short range organization of the paraffinic chains;

2) small angles $\left(s \simeq(30 \AA)^{-1}\right)$, where the scattering is due to the long range organization of the amphiphilic aggregates.

THE HIGH ANGLE REGION FOR TYPES I AND II. Around $s=(4.5 \AA)^{-1}$ the diagramms show a diffuse ring, similar to that of liquid paraffins, which characterizes the chaotic, short range organization of the methylene groups of the paraffinic chains [10]. Interestingly enough in the case of oriented samples the distribution of intensity along this ring depends 
upon the chosen configuration. It is isotropic when the beam is parallel to the director, it is intensified along the equator, which is also the direction of the alining magnetic field, when the beam is perpendicular to the director. Microdensitometer traces of the radial intensity distributions, along the equator and normal to it, are shown in figure 2. The anisotropy of the diffracted intensity in the perpendicular configuration indicates that the chains are not fully disordered, as in the case of an ordinary lyotropic system where the chains have a persistance length which exceeds that of a random conformation and where an average stretching axis (m) may be defined for each chain. The intensification of the diffraction in the equatorial plane, which contains the magnetic field, shows that $m$ is preferentially perpendicular to the field irrespective of the phase type.

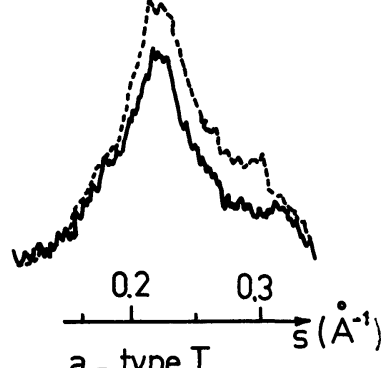

a - type I

... equatorial

- meridional
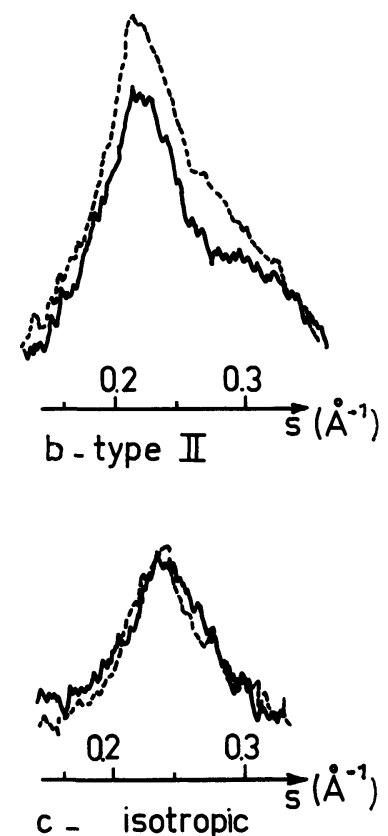

Fig. 2. - Microdensitometer analysis of the radial profiles of the rings of diffraction due to the paraffinic chains. The dotted lines represent sections along the equatorial plane, which contains the alining magnetic field; the full lines represent sections normal to the field, along the meridian.

The SMALl ANGLE REgION FOR TYPE II. - The densitometric mappings of the diffraction patterns [19] for the parallel and perpendicular configuration are shown in figure 3. First, $\mathbf{n}$, appears as the symmetry axis of the structure in the diffraction experiment as well as in the NMR one. Second, the pattern consists of rather wide streaks in the region of $s$ between $(10 \AA)^{-1}$ and $(100 \AA)^{-1}$. Superficially these streaks have important widths, as any disordered liquid, and their anisotropies in the perpendicular configuration are reminiscent of the patterns of thermotropic nematics without translational order [11]

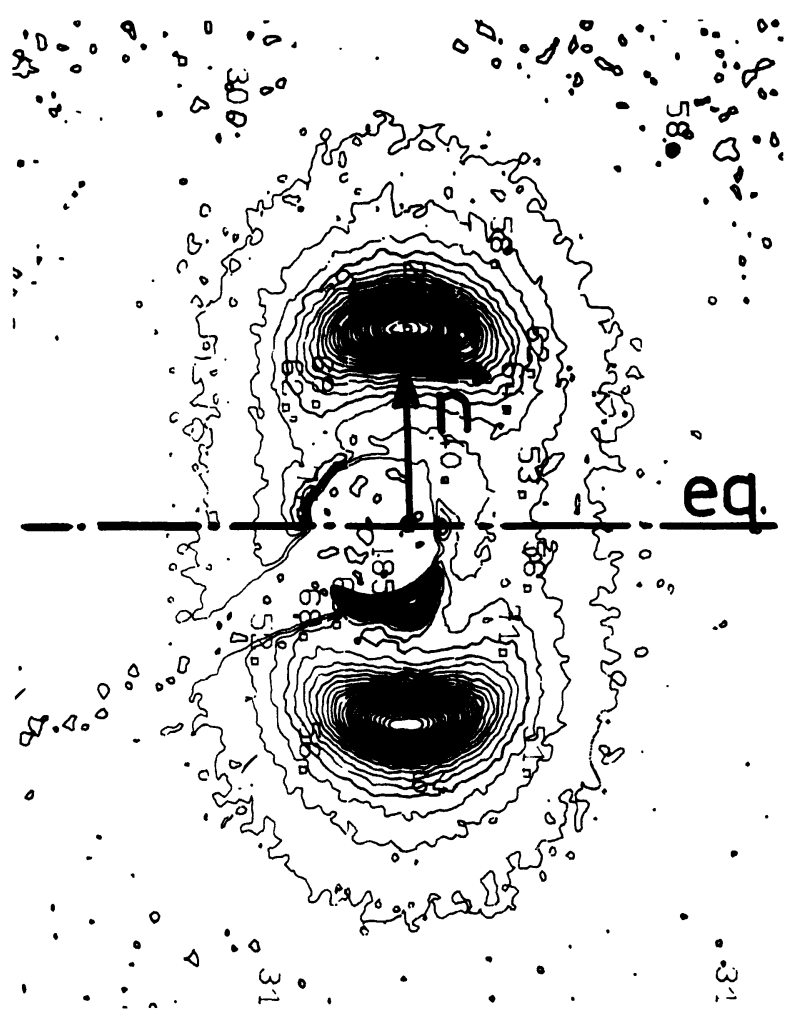

a_X-ray $\perp \vec{n}$

$\stackrel{0.234}{\longrightarrow} 10^{2} \mathrm{~s}\left(\mathrm{~A}^{-1}\right)$

b_ X-ray // $\vec{n}$

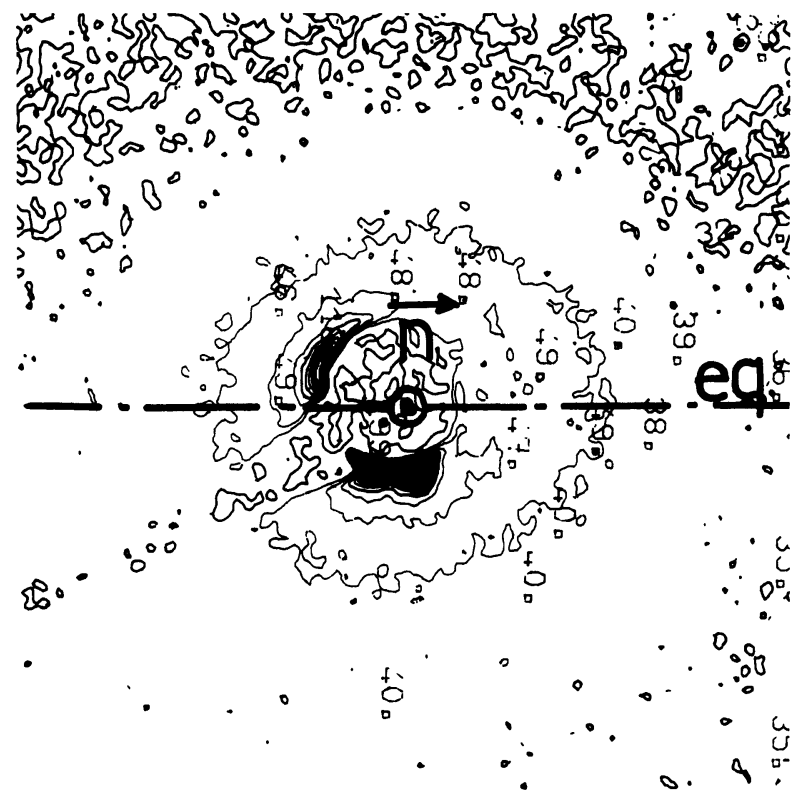

Fig. 3. - Microdensitometer analysis of the small angle diffraction patterns for a type II sample in perpendicular $(a)$ and parallel $(b)$ configurations. The X-ray beam is normal to the plane of the figure, the intersection of this plane with the equatorial plane which contains the magnetic field is labelled (eq.). The density scaling goes from 0 to 256 , the magnitude between principal lines is 32 and there are 3 intercalar lines. The beam stop, visible in the center and the low left corner, is a bit too small for the direct beam resulting in the high intensity spots in the centers of the diagrams. 
but the values of $s$ are smaller. On the other hand, the values of $s$ at the intensity maxima are quite in the range of those deduced from the peak positions in the diagramms of ordinary lyotropics with long range order [7]. This definitively suggests that the lyotropic nematic phases are assemblies of more or less well defined molecular aggregates of the lyotropic type with orientational order but without long range translational order.

In figure $3 a$ (perpendicular configuration) one can observe two intense wide spots along $\mathbf{n}$ at about $s=0.028 \AA^{-1}$ which are joined by two weaker streaks parallel to $\mathbf{n}$ at about $s=0.015 \AA^{-1}$. In figure $3 b$ (parallel configuration) one observes a ring the radius of which is about $0.014 \AA^{-1}$. These patterns are sections of the reciprocal space parallel and perpendicular to $\mathbf{n}$ respectively and the structure in this space may be schematized as an elongated, hollow, circular cylinder with an axis coincident with n. If one assumes, as a first approximation, that the diffraction is dominated by interferences between objects [12], the diffracting unit in real space is, via Fourier transformation, a flattened cylinder, or disc, with its major axis parallel to n. From the $s$ values quoted above the thickness of the unit would be about $37 \AA$ and its diameter $72 \AA$.

In analogy with ordered lyotropics the analysis of the diffraction data can proceed by considering the restrictions imposed on the diffracting unit : it contains a paraffinic aggregate and water with their volume ratio corresponding to the composition of the phase, one dimension of the paraffinic object can not exceed the thickness of a $C_{10}$ bilayer, about $20 \AA$ [13]. Under these restrictions the amphiphilic aggregate might be described as a disc, or oblate ellipsoid, with a thickness of about $20 \AA$ and a diameter of about $65 \AA$ [14]. The different intensity distributions along $\mathbf{n}$ and parallel to $\mathbf{n}$ in figure $3 a$ suggest that in the type II phase the discs tend to associate with their major axes $\left(a_{\mathrm{d}}\right)$ parallel thus facilitating their close packing.

The SMALl ANGLE REgion FOR TYPE I. - The densitometric mappings of the diffraction patterns [19] for the parallel and perpendicular configurations are shown in figure 4. As for type II $\mathbf{n}$ appears as the symmetry axis of the structure in the diffraction experiment as well as in the NMR experiment, there is also no long range order.

In figure $4 a$ (perpendicular configuration) one can observe two intense spots along $\mathbf{n}$ at about $s=0.026 \AA^{-1}$. They are not joined by parallel streaks as was the case for type II (the intense equatorial scattering visible at the left side of the beam stop is an artefact : part of the direct beam is not absorbed by the beam stop). In figure $4 b$ (parallel configuration) one observes a ring the radius of which is about $0.025 \AA^{-1}$, larger than that of the ring observed for type II. The distance between the spots
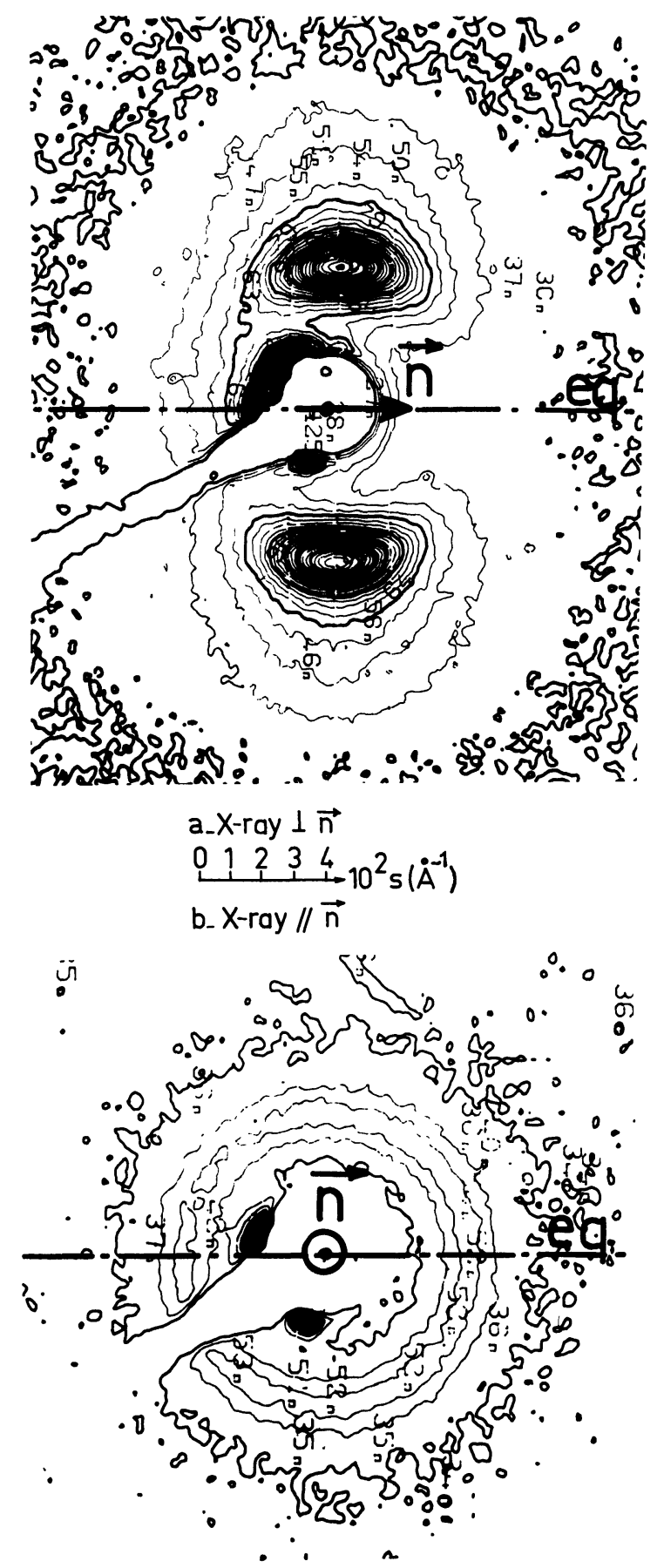

Fig. 4. - Microdensitometer analysis of the small angle scatterings for a type I sample in the perpendicular and parallel configurations. Same conditions as figure 3 .

in figure $4 a$ coincides with the diameter of the ring in figure $4 b$ and this suggests that the reciprocal structure is a torus with its major axis parallel to $\mathbf{n}$. Via Fourier transformation the diffracting unit in the real space is therefore an elongated cylinder with its major axis parallel to $\mathbf{n}$ and with an average diameter of $38.5 \AA$. In analogy with the hexagonal structures of the classical lyotropic mesophases [13] the amphiphilic aggregate might be described as a 
cylinder or a prolate ellipsoid, with average diameter of about $26 \AA$ and with its major axis $\left(a_{\mathrm{c}}\right)$ parallel to $\mathbf{n}$ [14]. As no equatorial spot is visible in the perpendicular pattern nothing can be said about the length of the cylinder. In the type I phase the cylinders tend to pack with their long axes parallel. Also, no evidence for translational order in the packing, lateral or longitudinal, has been detected so far.

Discussion. - Changes in the composition of a nematic lyotropic mixture induce a change in sign of $\Delta \chi$. Concomitantly, structural changes occur in the phase. First the high angle X-ray intensity distributions for both type I and type II phases show that $\mathbf{m}$, the stretching vector of the chain, is preferentially aligned perpendicular to the field. Hence the striking inversion of $\Delta \chi$ can not be correlated with a change of orientation at the molecular level. As a matter of fact, studies of single crystals of analogue amphiphiles yield $\Delta \chi_{m}<0$ for a single molecule [15]. We would infer here a reduced value of $\Delta \chi_{m}$ due to conformational averaging but, nevertheless, because of the residual elongation of the chain a negative $\Delta \chi_{\mathrm{m}}$. Consequently we anticipate the isolated amphiphiles to exhibit a propensity to align $\mathbf{m}$ normal to the magnetic field independent of the phase type I or II. And, when we consider the equilibrium orientation of the proposed aggregate structures in the field we find that this molecular orientational preference is preserved (ignoring edge and end effects).

Second, the low angle intensity distributions demonstrate that the primary difference between type I and type II phases is associated with the shapes of the structural elements in the phases. Prolate in type I, oblate in type II, the aggregates orient in the field so that their major axes $a_{\mathrm{d}}$ and $a_{\mathrm{c}}$ are parallel and perpendicular, respectively, to the field. It is of interest to note that these equilibrium orientations are those expected from the magnetic shape anisotropies of homogeneous prolate and oblate objects in an uniform magnetic field [16].

Presumably, as in ordinary lyotropics, the amphiphile axes are on average normal to the amphiphilewater interface [17]. Therefore, in lyotropic nematics, both the molecular and shape magnetic anisotropies have convergent orienting effects resulting in an alignment of most of this interface parallel to the field.

In conclusion, the magnetic and diffraction properties associated with the transition from type II to type I lyotropic nematics can be accounted for with a change in the shape of the elemental structural unit of the phase. No unusual behavior of the amphiphile molecules, nor substantial deviation from their usual mode of local aggregation in lyotropic liquid crystals need be postulated at this transition. Lastly one must indicate that the preferences for homeotropic and planar alignments for type II and I respectively are also coherent with this model [18].

Acknowledgments. - We are grateful to L. Liébert for his interest and help in the early stages of this work and to Y. Hendrikx for critical advice on the phase diagrams. One of us (E.T.S.) wishes to acknowledge the DRET (grant $n^{\circ}$ 75-390) for financial support.

\section{References and notes}

[1] Lawson, K. D. and Flautt, T. J., J. Am. Chem. Soc. 89 (1967) 5490.

[2] Radley, K. and Saupe, A., Mol. Cryst. Liq. Cryst. 44 (1978) 227.

[3] Khetrapal, C. L., Kunwar, A. C., Tracey, A. S. and DIEHL, P., Lyotropic liquid crystals NMR basic principles and progress, Vol. 9, P. Diehl, E. Fluck and R. Kosfeld, Editors (Springer-Verlag, Berlin) 1975.

[4] Liébert, L., Martinet, A. et Charvolin, J., Système liquide anisotrope dont les propriétés varient sous l'action d'un champ magnétique faible. Brevet ANVAR no 7825744 7 sept. (1978)

[5] Liébert, L., Martinet, A., J. Physique Lett. 40 (1979) L-363.

[6] Chen, D. M., Funiwara, F. Y. and Reeves, L. W., Can. J. Chem. 55 (1977) 2396.

[7] Charvolin, J. and Tardieu, A., Solid State Physics, edited by F. Seitz and D. Turnbull (Academic Press) 1978, suppl. 14.

[8] Under similar experimental conditions thermotropic nematics are also distorted and walls, without any periodic organization, are observable. For a periodic organization to appear the distortion of the director has to be contraried by some intrinsic energy term. We may quote in this respect the roles of a flexo-electric coupling, of an hydro- dynamic backflow in nematics, of the layer arrangements in smectics and cholesterics.

[9] Comes, R., Lambert, M. and Guinier, A., Acta Cryst. A 26 (1970) 244.

[10] Luzzati, V., Mustacchi, M., Skoulios, A. et Husson, F., Acta Cryst. 13 (1960) 660.

[11] Chistyakov, I., Adv. Liq. Cryst. 1 (1975) 143 (Acad. Press)

[12] The relative contributions to the intensity of the structure factor of the aggregates and of the interferences between aggregates is an important point for an accurate description of the structure but not essential to account for the relation between $\Delta \chi$ and the mode of aggregation presented here. We assume that the interferences between aggregates dominate and their mean distance of approach, along every direction, determine the diffracting unit.

[13] Gallot, B. et Skoulios, A., Kolloid Z. and Z. Polymer. 208 (1966) 37.

[14] The diffraction data are ambiguous with respect to the topology of the structure, paraffin in water, or vice versa. As the volume ratio of water and amphiphilic molecules is about one, this uncertainty is difficult to remove at the moment. We decide in favor of a structure with a continuous aqueous medium because we can infer from closely related phase diagrams, that the lyotropic nematic 
phases are close to hexagonal and micellar phases of this type.

Ekwall, P., Liq. Cryst. 1 (1975) 1 (Acad. Press).

[15] Londsale, K., Proc. R. Soc. (London) A 171 (1939) 541.

[16] LaNDAU, L. D. and LifShITZ, E. M., Electrodynamics of continuous media, p. 169-170, $42-45$ (Pergamon Press) 1960.

[17] Charvolin, J., Manneville, P. and Deloche, B., Chem. Phys. Lett. 23 (1973) 345.
[18] NMR experiments have shown that infinite bilayers and cylinders tend to aline parallel to glass plates spontaneously; similar alignments would be anticipated for finites discs and cylinders.

Mely, B. and Charvolin, J., Chem. Phys. Lipids 15 (1975) 161

[19] We have obtained the densitometric mappings of the diffraction patterns from the Service de Microdensitométrie $d u$ C.N.R.S. (Orsay). 